\title{
Pure transgastric NOTES in an adnexal procedure: the first human case report
}

Natural orifice transluminal endoscopic surgery (NOTES) is an innovative procedure that represents a further evolution of minimally invasive surgery. To date, there are few cases reported in the literature that have been performed using pure NOTES [1-3]. Recently, Lee et al. [4] described pure transvaginal NOTES in adnexal procedures using modern laparoscopic rigid instruments. To the best of our knowledge, however, transgastric NOTES for adnexal disease has not previously been reported in humans. Herein, we report on a pure transgastric NOTES procedure performed for adnexal disease in a young woman.

A 36-year-old woman presented to our hospital with left lower abdominal pain for 3 days. Her serum beta-human chorionic gonadotropin (beta-hCG) was $547.23 \mathrm{mIU} / \mathrm{mL}$ (normal <5 mIU $/ \mathrm{mL}$ ). Transvaginal ultrasonography confirmed the diagnosis of a left tubal ectopic pregnancy and right simple ovarian cyst.

A pure transgastric NOTES procedure was performed after approval by the hospital ethics committee. The stages of the operation we performed were as follows: (i) creation of gastric access using a percutaneous endoscopic gastrostomy (PEG)like technique; (ii) establishment of a pneumoperitoneum with a laparoscopic insufflator; (iii) visualization of the uterus ( Fig. 1 a) and inspection of the adnexa, which revealed a superficial endometriosis lesion on the right ovarian wall, in addition to the left fallopian tubal ectopic pregnancy mass ( Fig.1b) and right ovarian cyst; (iv) cystotomy of the ovarian cyst with an injection needle; (v) electrocautery of the endometriosis lesion with a Coagrasper; (vi) transgastric NOTES salpingostomy ( Fig. 1 c) and dissection of the ectopic pregnancy lesion from the tubal wall without laparoscopic assistance; (vii) removal of the lesion and confirmation that no remnant was left; (viii) closure of the gastric incision with endoclips and a nylon loop ( Fig. 1 d).

The ectopic pregnancy lesion was removed successfully, giving a specimen of $4.0 \times 2.0 \mathrm{~cm}$ ( Fig. 2). The patient did well postoperatively and had no complications. Her serum beta-hCG returned to normal 3 days after the operation. Histo-
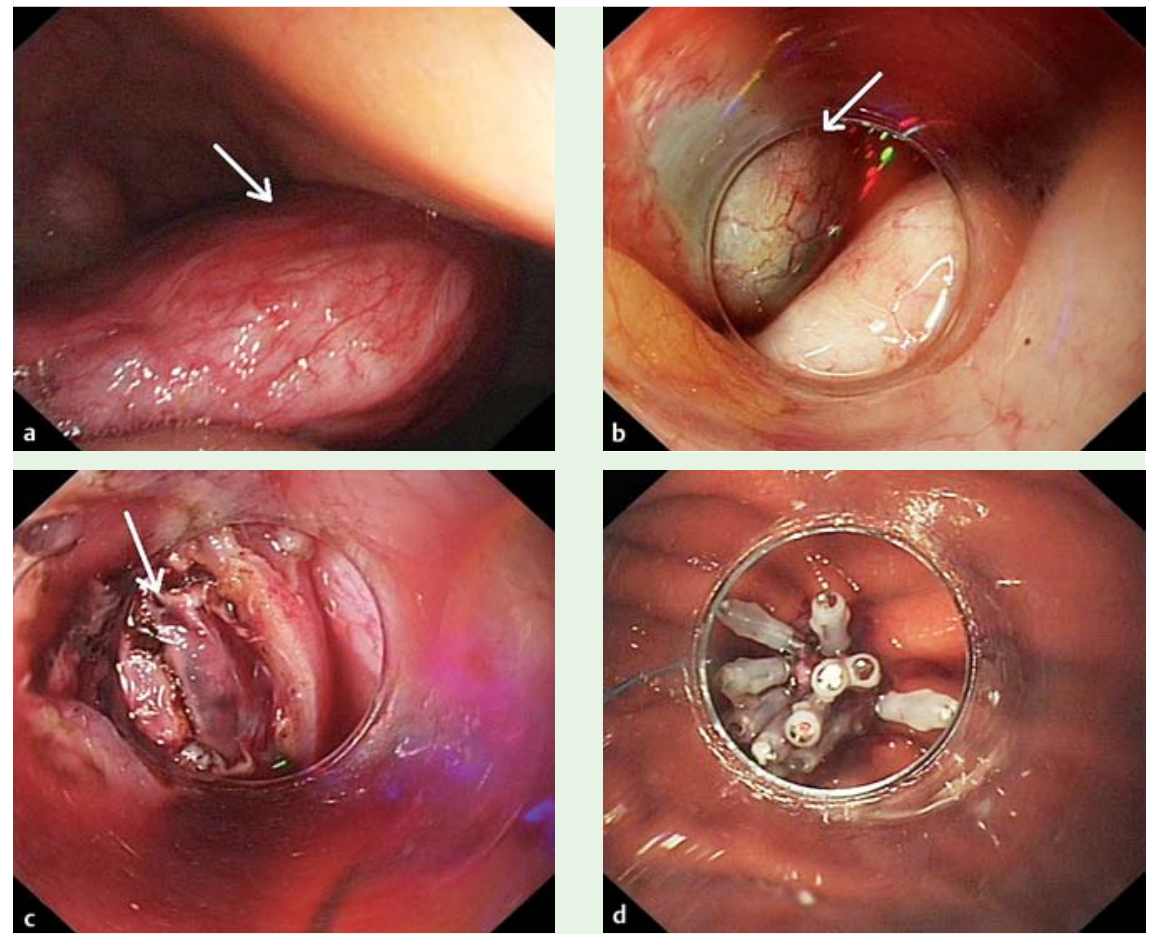

Fig. 1 Endoscopic views during pure natural orifice transluminal endoscopic surgery (NOTES) in a woman with a left tubal ectopic pregnancy showing: $\mathbf{a}$ the uterus (arrow); $\mathbf{b}$ the left fallopian

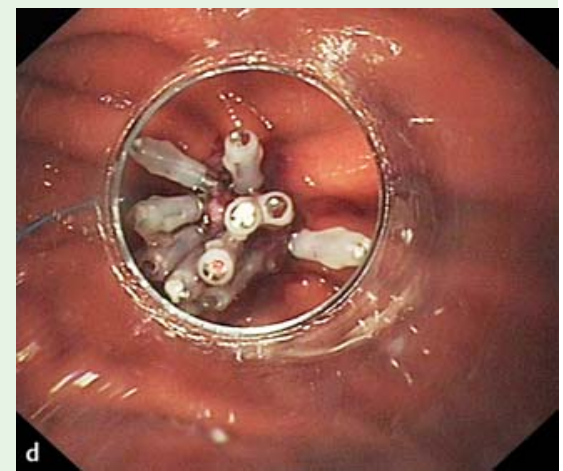

tubal mass with purplish-black discoloration (arrow); $\mathbf{c}$ the lesion seen through tubal incision (arrow); $\mathbf{d}$ the completed gastric closure after nylon-loop ligation and placement of endoclips.

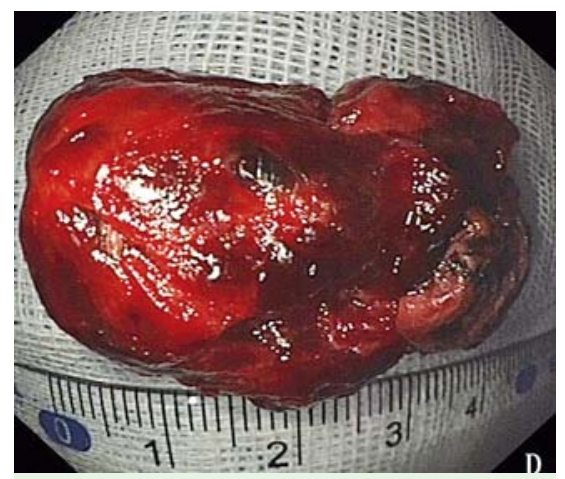

Fig. 2 Photograph of the embryonic tissue following removal.

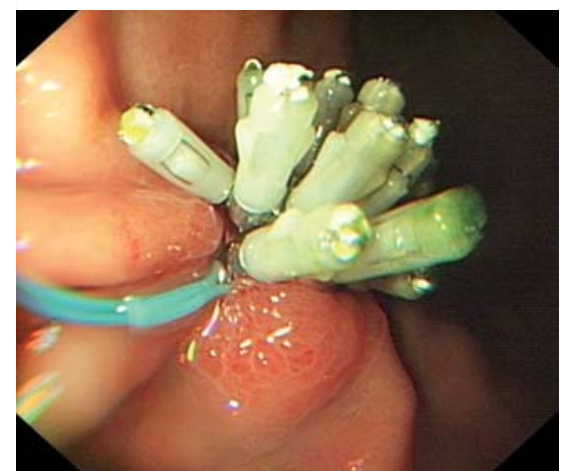

Fig. 3 Endoscopic view of the stomach 5 days later showing the well-healed gastric incision.

\section{Video 1}

logical examination confirmed the presFollow-up endoscopy 5 days later showed good healing of the gastric incision (๑ Fig.3).

\section{Endoscopy_UCTN_Code_TTT_1AT_2AF}

Competing interests: None
Views of a pure transgastric natural orifice transluminal endoscopic surgery (NOTES) procedure performed in a young woman with a tubal ectopic pregnancy showing the stages involved in the successful salpingostomy of the ectopic pregnancy, ovarian cystotomy, and electrical cautery of an endometriosis lesion.

the 
B.-R. Liu" ${ }^{1, ~ *, ~ X .-C . ~ K o n g ~}{ }^{2, ~ *, ~ G .-X . ~ C u i ~}{ }^{1}$, X.-Y. Zhang ${ }^{1}$, J.-T. Song ${ }^{1}$, Y. Kuang ${ }^{2}$, L.-J. Kong ${ }^{1}$, Y. Si ${ }^{2}$

${ }^{1}$ Department of Gastroenterology and Hepatology, The Second Affiliated Hospital of Harbin Medical University, Harbin, China

2 Department of Gynecology and Obstetrics, The Second Affiliated Hospital of Harbin Medical University, Harbin, China

* B.-R. Liu and X.-C. Kong contributed equally to this article.

\section{References}

1 de Sousa LH, de Sousa JA, de Sousa Filho LH et al. Totally NOTES (T-NOTES) transvaginal cholecystectomy using two endoscopes: preliminary report. Surg Endosc 2009; 23: 2550-2555

2 Bessler M, Gumbs AA, Milone L et al. Pure natural orifice transluminal endoscopic surgery (NOTES) cholecystectomy. Surg Endosc 2010; 24: 2316-2317

3 Coomber RS, Sodergren MH, Clark J et al. Natural orifice translumenal endoscopic surgery applications in clinical practice. World J Gastrointest Endosc 2012; 4: 65 - 74

$4 \mathrm{Lee} C L, W u K Y, S u H$ et al. Transvaginal natural orifice transluminal endoscopic surgery (NOTES) in adnexal procedures. J Minim Invasive Gynecol 2012; 19: 509-513

\section{Bibliography}

Dol http://dx.doi.org/

10.1055/s-0033-1344559

Endoscopy 2013; 45: E290-E291

(c) Georg Thieme Verlag KG

Stuttgart · New York

ISSN 0013-726X

\section{Corresponding author}

B.-R. Liu, MD, PhD

Department of Gastroenterology and Hepatology The Second Affiliated Hospital of Harbin Medical University

246 Xue-fu Road

Nan-gang District

Harbin 150086

China

Fax: +86-451-86605980

13704510648@126.com 